\title{
The adaptor protein TRADD activates distinct mechanisms of apoptosis from the nucleus and the cytoplasm
}

\author{
LM Bender ${ }^{1}$, MJ Morgan², LR Thomas ${ }^{1}$, Z-G Liu ${ }^{2}$ and \\ A Thorburn*,1,3 \\ 1 Department of Cancer Biology, Wake Forest University School of Medicine, \\ Medical Center Blvd, Winston-Salem, NC 27157, USA; \\ 2 The National Institute of Health, National Cancer Institute, Center for Cancer \\ Research, Bethesda, MD 20892, USA; \\ ${ }^{3}$ Department of Pharmacology, University of Colorado Health Sciences Center, \\ Fitzsimmons Campus, PO Box 6511, Mail Stop 8303, Aurora, CO 80045, USA \\ * Corresponding author: A Thorburn, Department of Pharmacology, University of \\ Colorado Health Sciences Center, UCHSC at Fitzsimmons, L18-6100 RC1 \\ South Tower, PO Box 6511, Mail Stop 8303, Aurora, CO 80045-0508, USA. \\ Tel: 303-724-3290; Fax: 303-724-3663; \\ E-mail: Andrew.Thorburn@UCHSC.edu
}

Received 30.6.04; revised 06.12.04; accepted 22.12.04; published online 11.3.05 Edited by $\mathrm{P}$ Vandenabeele

\begin{abstract}
TNFR1 associated death domain protein (TRADD) contains an $\mathrm{N}$-terminal TRAF binding domain and a $\mathrm{C}$-terminal death domain along with nuclear import and export sequences that cause shuttling between the cytoplasm and nucleus. The death domain of TRADD contains the nuclear import sequence and expression of the core death domain (nuclear TRADD) results in exclusive nuclear localization and activation of a distinct apoptotic pathway. Cytoplasmic TRADD activates apoptosis through Fas-associated death domain protein (FADD) and caspase-8 activation that was blocked by caspase inhibitors or dominant-negative FADD. These inhibitors did not inhibit death induced by nuclear TRADD, which could only be inhibited by combining caspase inhibitors and a serine protease inhibitor. The pathway activated by nuclear TRADD requires caspase-9 catalytic activity. However, apoptosis activating factor deficiency confers only partial protection from death. This pathway represents an alternate means by which TRADD can regulate cell death independently of FADD and caspase-8 that occurs from the nucleus rather than the cytoplasm.
\end{abstract}

Cell Death and Differentiation (2005) 12, 473-481.

doi:10.1038/sj.cdd.4401578

Published online 11 March 2005

Keywords: TRADD; FADD; caspase; serine protease; nuclear localization

Abbreviations: AEBSF, (4-(2-Aminoethyl)benzenesulfonyl fluoride hydrochloride); APAF-1, apoptosis activating factor I; FADD, Fas associated death domain protein; FADD-DD, dominant-negative FADD; IAP, inhibitor of apoptosis protein; ICR-191, 6-Chloro-9[3-(2-chloroethylamino)propylamino]-2-methyoxyacri- dine; MEFs, mouse embryonic fibroblasts; siRNA, short interfering RNA; PARP, poly(ADP-ribose) polymerase; Stat-1, signal transducer and activator of transcription I; TNFR, TNF receptor; TRADD, TNFR1 associated death domain protein; YFP, yellow fluorescent protein; zVAD.fmk, $N$-Benzyloxcarbonyl-ValAla-Asp(o-Me) fluoromethyl ketone

\section{Introduction}

There are two well-characterized apoptotic pathways in mammalian cells that activate initiator caspases, which then cleave and activate effector caspases to disassemble the cell. $^{1,2}$ Death receptor-induced apoptosis requires ligand binding to one of the six known death receptors. ${ }^{3,4}$ Ligand binding is thought to induce a conformational change in the cytoplasmic domain of the receptor that facilitates recruitment of adaptor proteins such as TNFR1-associated death domain protein (TRADD) or Fas associated death domain protein (FADD) to the receptor. TRADD and FADD then recruit and activate the initiator caspase, caspase-8, to ultimately result in effector caspase activation, and apoptosis. Stress pathways, on the other hand, cause release of mitochondrial proteins including cytochrome $c$ into the cytosol. Cytoplasmic cytochrome $c$ forms a complex with apoptosis activating factor (APAF-1), pro-caspase-9, and dATP generating the apoptosome. The initiator caspase-9 becomes activated when the apoptosome is formed, and caspase-9 then activates effector caspases to promote apoptosis. Death receptors can also activate the mitochondrial-dependent apoptotic pathway by caspase-8 mediated cleavage of the proapoptotic $\mathrm{Bcl} 2$ protein Bid. ${ }^{5}$ Cleaved Bid translocates to the mitochondria to facilitate cytochrome $c$ release and caspase-9 activation.

Other proteases also regulate caspase activation and apoptosis. ${ }^{6,7}$ For example, cathepsins have been implicated in the activation of caspases and in causing apoptosis-like death in the absence of caspase activity. ${ }^{8}$ In addition, the mitochondrial serine protease Omi/HtrA2 has been shown to facilitate caspase-9 activation by disrupting inhibitor of apoptosis protein (IAP) inhibition of caspase- $9^{9-11}$ and degrading IAPs. ${ }^{12,13}$

TNF signals through two receptors, tumor necrosis factor receptor (TNF-R) 1 and TNF-R2. Signaling through TNF$\mathrm{R} 1^{14,15}$ activates both prosurvival signals including $\mathrm{NF}_{\kappa} \mathrm{B}$ and Jun $\mathrm{N}$-terminal kinase as well as proapoptotic signals. Signaling downstream of TNF-R1 requires the adaptor protein TRADD, which functions by recruiting other proteins to the active receptor complex. TRADD is comprised of two distinct functional domains including an $\mathrm{N}$-terminal domain that mediates binding to TRAF2 and promotes $\mathrm{NF}_{\kappa} \mathrm{B}$ activation, and a C-terminal death domain that is required for binding to death domain containing proteins such as FADD and Rip. TRADD binding to FADD, which has been suggested to 
occur in the cytoplasm after dissociation from the receptor complex ${ }^{16}$ and more recently proposed to occur in endocytic vesicles, ${ }^{17}$ activates caspase-8-dependent apoptosis. Surprisingly, for a protein that binds to a cell surface receptor and signals from the cytoplasm or endocytic vesicles, TRADD also contains a nuclear import sequence and a nuclear export sequence that together promote nuclear/cytoplasmic shuttling. Expression of full-length TRADD in the presence of the CRM-1-dependent nuclear export inhibitor leptomycin B traps TRADD in the nucleus and activates an apoptotic pathway that depends upon this alternate subcellular localization. ${ }^{18}$ Expression of the core helices of the death domain aa 222289 (nuclear TRADD), which contains the nuclear import sequence but no export sequence, results in an exclusively nuclear localized protein that kills cells when it is in the nucleus but cannot kill cells when it is forced outside the nucleus. ${ }^{18}$ As the established FADD and caspase-8-dependent pathway occurs in the cytoplasm, we hypothesized that apoptosis induced by nuclear TRADD may occur by a different mechanism. Here, we test this hypothesis by dissecting the apoptosis pathway that is activated by an exclusively nuclear TRADD mutant and comparing it with the pathway that is activated by an exclusively cytoplasmic TRADD mutant. We found that while cytoplasmic TRADD kills cells via FADD and caspase-8, the nuclear pathway is independent of caspase-8 and FADD. Instead, nuclear TRADD kills cells through caspase-9. These data show that TRADD can induce apoptosis via two distinct pathways, one requiring caspase8 and FADD, which works in the cytoplasm, and the other relying upon caspase- 9 activation and the nuclear localization of the protein.

\section{Results}

\section{Nuclear TRADD-induced death is caspase-8 and FADD-independent}

The only characterized mechanism by which TRADD can induce apoptosis involves FADD and caspase-8 recruitment, which is thought to occur in a cytoplasmic complex ${ }^{16}$ or endocytic vesicles. ${ }^{17}$ However, we previously showed that $\mathrm{N}$-Benzyloxcarbonyl-Val-Ala-Asp(o-Me) fluoromethyl ketone (zVAD.fmk), which inhibits caspase-8-dependent apoptosis, was unable to block cell death induced by a nuclear TRADD molecule, ${ }^{18}$ suggesting that nuclear TRADD works independently of these proteins. To test this hypothesis, we expressed a truncated TRADD death domain molecule that retains the nuclear import sequence but has no nuclear export sequence and is exclusively nuclear ${ }^{18}$ (nuclear TRADD) or a TRADD mutant that is exclusively in the cytoplasm (cytoplasmic TRADD) through the addition of a myristolation signal that anchors it to the cell membrane preventing nuclear shuttling (Figure 1a). These molecules were coexpressed along with FADD-DD (dominant-negative FADD) to test if FADD was required for cell death. As predicted, death induced by cytoplasmic TRADD was blocked by FADD-DD, but death induced by nuclear TRADD was not (Figure 1b). As a complementary way to test if death induced by nuclear TRADD was independent of FADD, FADD-deficient mouse embryonic fibroblasts (MEFs) were used. These cells were injected with yellow fluorescent protein (YFP) or YFP-nuclear TRADD. The wild type and FADD-deficient MEFs were equally sensitive to nuclear TRADD-induced death indicating that FADD was not required for activation of the apoptotic pathway by nuclear TRADD (Figure 1c). Although FADD can be localized to the nucleus, ${ }^{19,20}$ the nuclear TRADD construct consists of only amino acids 222-289, which is smaller than the region of the protein (aa 195-312) that was previously reported to be required for FADD-TRADD interaction. ${ }^{21}$ We therefore tested if nuclear TRADD could interact with FADD in a directed two hybrid. Figure 1d shows that while FADD can interact with the full-length TRADD molecule that was anchored to the membrane to produce the solely cytoplasmic TRADD mutant, FADD cannot interact with the nuclear TRADD construct in yeast. These data show that while the cytoplasmic TRADD molecule can bind to FADD and kills cells through an FADD-dependent mechanism, the nuclear TRADD construct consisting of only the core helices of the death domain neither interacts with FADD nor kills cells through a FADD-dependent mechanism.

To test if caspase-8 was required for nuclear TRADDinduced cell death, we overexpressed a catalytically inactive mutant of caspase-8 (DN-Caspase-8) in the presence of the TRADD mutants in HeLa cells. As expected, DN-caspase-8 inhibited cytoplasmic TRADD-induced death. In contrast, nuclear TRADD-induced death was not inhibited by DN caspase-8 expression (Figure $2 \mathrm{a}$ ). To more definitively test the role of caspase-8, caspase-8-deficient BJAB cells were used. BJAB cells were mutagenized with ICR191 and TRAIL resistant clones were selected. The clones were screened by immunoblotting for caspase-8 expression (Figure $2 b$, inset). After identifying a caspase-8 deficient clone, the wild type or caspase-8 deficient BJABs were transiently transfected with YFP-nuclear TRADD, GFP-cytoplasmic TRADD, or YFP. At 24-30 h after transfection, cells were counted and scored as either viable or death based upon apoptotic morphology. When compared with parental cells, caspase- 8 deficient cells were resistant to cytoplasmic TRADD, but were equally sensitive to nuclear TRADD-induced death (Figure 2b).

\section{Expression of nuclear TRADD activates both caspase-dependent and caspase-independent apoptotic pathways}

We previously reported that death induced by nuclear TRADD could not be completely blocked by the caspase inhibitor zVAD.fmk. ${ }^{18}$ However, these data did not exclude the possibility that caspase activation occurred in response to nuclear TRADD expression. To test if death induced by nuclear TRADD was associated with caspase activation, we assessed caspase- 3 processing in cells transfected with either nuclear or cytoplasmic TRADD (Figure 3a). Both TRADD mutants caused the appearance of processed caspase-3. We next assessed poly(ADP-ribose) polymerase (PARP) cleavage and found that expression of both nuclear and cytoplasmic TRADD produced an $89 \mathrm{kDa}$ PARP fragment that is characteristic of caspase activation. This cleavage event was inhibited by zVAD.fmk (Figure 3a). Caspase- 9 catalytic activity was next measured in cells transfected with 
YFP control, nuclear TRADD or cytoplasmic TRADD. Both TRADD molecules caused caspase- 9 activation (Figure $3 b$ ). These data indicate that nuclear TRADD can activate caspases.

A number of other proteases including Omi/HtrA2, cathepsins and various serine proteases regulate apoptosis in response to various stimuli. ${ }^{6-8}$ To clarify the potential involvement of other proteases in nuclear TRADD-induced death, the broad spectrum serine protease inhibitor AEBSF ((4-(2-Aminoethyl)benzenesulfonyl fluoride hydrochloride)) was tested for the ability to inhibit nuclear TRADD-induced apoptosis. HeLa cells were microinjected with the YFP-tagged nuclear TRADD and cytoplasmic TRADD constructs. At $4 \mathrm{~h}$ after injection, the cells were treated with vehicle, the caspase

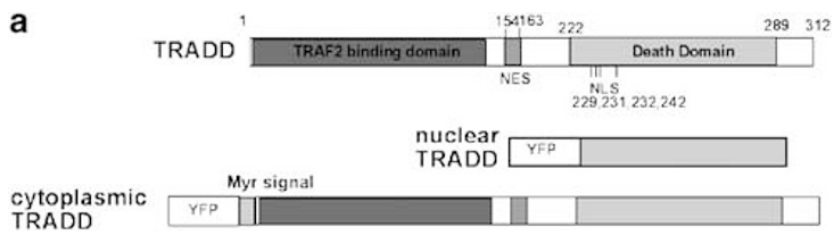

b

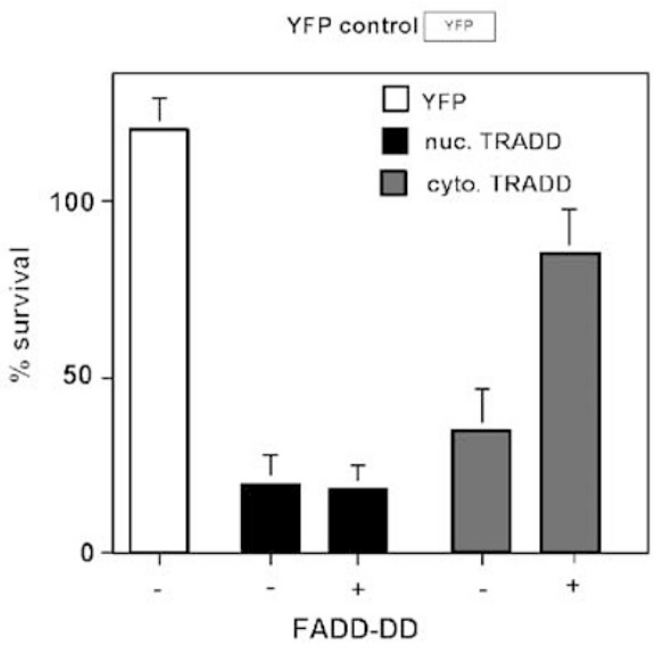

C

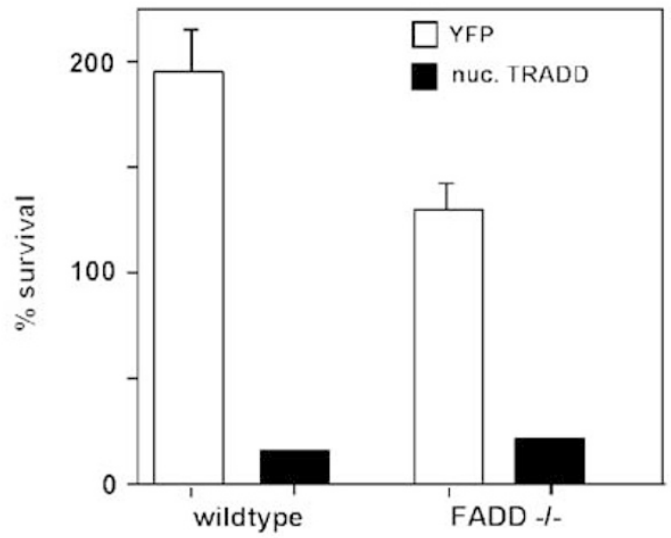

d

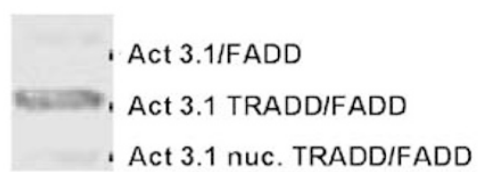

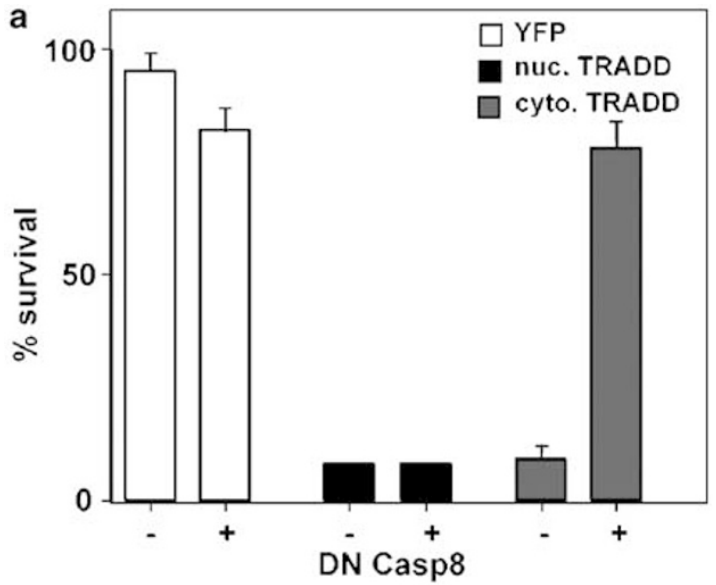

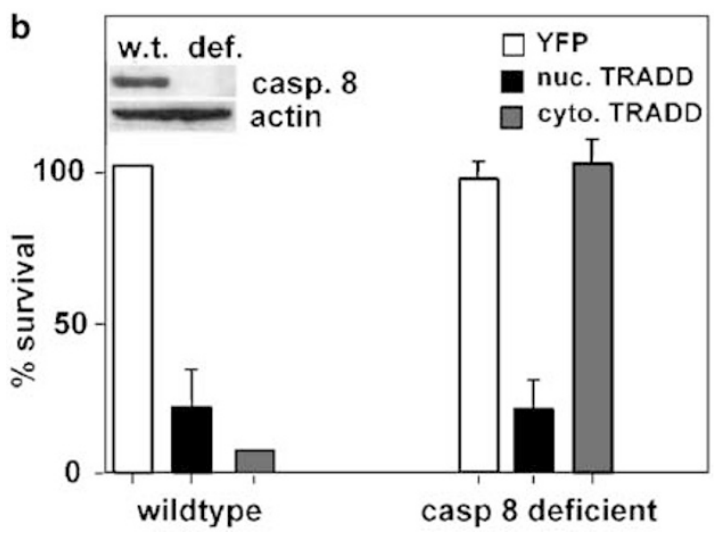

Figure 2 Nuclear TRADD-induced death is independent of caspase-8. (a) HeLa cells were microinjected with YFP-tagged nuclear TRADD, cytoplasmic TRADD, or YFP in the presence of either empty vector (-) or DN-caspase-8 expression plasmid $(+)$. The percent survival of fluorescent cells was determined by microscopy indicating that dominant-negative caspase-8 inhibited cell death caused by cytoplasmic TRADD but not nuclear TRADD. (b) Wild-type BJAB cells and BJAB cells deficient in caspase- 8 were characterized by Western blotting (inset) and transiently transfected with YFP-nuclear TRADD, YFPcytoplasmic TRADD, or YFP. At $24 \mathrm{~h}$ after injection, green cells were counted and scored as either viable or dead based upon morphology, indicating that caspase8 deficient cells are sensitive to nuclear TRADD-induced death but resistant to cytoplasmic TRADD

Figure 1 Nuclear TRADD kills cells independently of FADD. (a) Schematic representation of constructs used. TRADD contains a death domain and a TRAFbinding domain along with a nuclear export sequence (amino acids 154-163) and a nuclear localization sequence (amino acids 229, 231, 232 and 242). Nuclear TRADD contains only the core death domain sequence with a YFP tag, cytoplasmic TRADD contains the full-length protein with an added myristylation membrane targeting sequence and a YFP tag, control experiments were performed with YFP alone. (b) HeLa cells were microinjected with YFP-tagged nuclear or cytoplasmic TRADD, or YFP alone in the presence of either empty vector, or an FADD-DD (dominant-negative FADD) expression plasmid. Fluorescent cells were assessed by microscopy to determine percent survival. Dominant-negative FADD inhibited cell death caused by cytoplasmic TRADD but not nuclear TRADD. (c) Wild type and FADD deficient MEFs were microinjected with YFP or YFP-nuclear TRADD. Both cell types died equally efficiently in response to nuclear TRADD. (d) pAct 3.1, pAct 3.1TRADD, or pAct 3.1 nuclear TRADD were co-transformed into the Y190 yeast strain with pGB14 FADD. Transformants were plated on selective media. Individual colonies were allowed to grow, and a blue-white $\beta$-Galactosidase filter lift assay was performed indicating that while FADD binds to full-length TRADD, FADD cannot interact with the nuclear TRADD mutant in yeast 
a

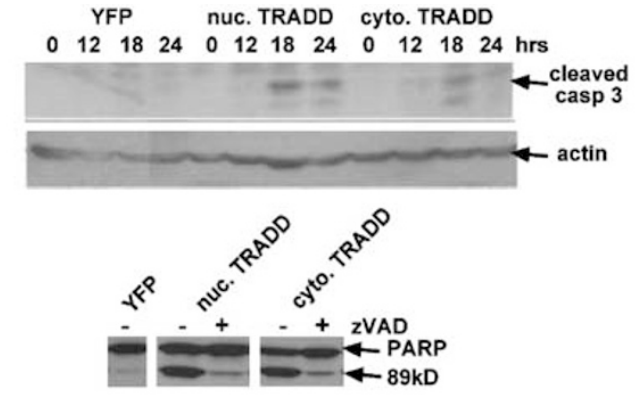

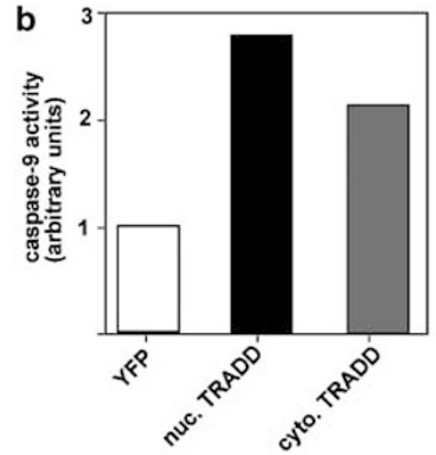

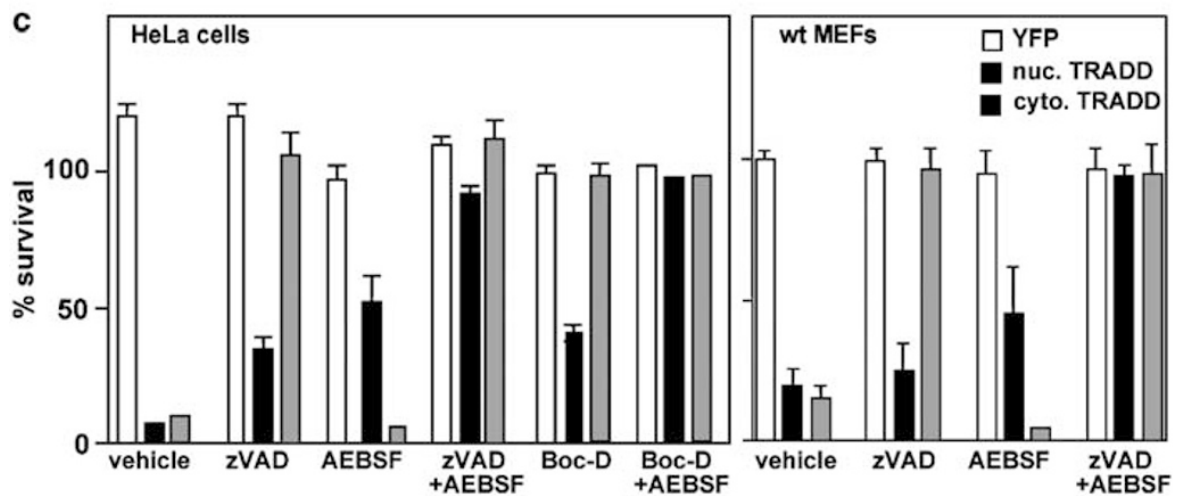

Figure 3 Nuclear TRADD expression activates caspase-dependent and serine protease-dependent cell death. (a) Upper panel, YFP, YFP-nuclear TRADD, or YFPcytoplasmic TRADD was transiently transfected into HeLa cells. Cells were harvested at the indicated times, lysed and immunoblotting was performed on whole-cell lysates with antibodies specific to cleaved caspase 3 , and $\beta$-actin indicating that caspase-3 is activated by both nuclear and cytoplasmic TRADD. Lower panel, lysates from transfected cells treated with or without the caspase inhibitor zVAD.fmk were harvested at $24 \mathrm{~h}$ and probed with an antibody that recognizes intact and caspasecleaved PARP. Both nuclear and cytoplasmic TRADD cause PARP cleavage to produce an $89 \mathrm{kDa}$ fragment that is blocked by caspase inhibition. (b) HeLa cells were transfected with YFP, nuclear TRADD or cytoplasmic TRADD, whole-cell lysates were harvested after $18 \mathrm{~h}$ and used in a caspase-9 activity assay. Both nuclear and cytoplasmic TRADD caused increased caspase activity. (c) HeLa cells (left panel) and wild-type MEFs (right panel) were microinjected with plasmid expressing YFP, YFP-nuclear TRADD or YFP-cytoplasmic TRADD. Cells were treated with caspase inhibitors zVAD.fmk or Boc-D or the serine protease inhibitor AEBSF or combinations of inhibitors as indicated and cell survival was determined after 18-24h. Cytoplasmic TRADD-induced death was blocked by caspase inhibition alone while nuclear TRADD-induced cell death was only partially blocked by each inhibitor alone but completely blocked by combined caspase and serine protease inhibition

inhibitors zVAD.fmk or Boc-D, AEBSF, or combination of caspase inhibitor plus AEBSF and the YFP expressing cells were counted. As expected, the exclusively cytoplasmic TRADD protein induces apoptosis through a mechanism that is entirely caspase dependent and was efficiently blocked by caspase inhibitors alone. In contrast, either caspase or serine protease inhibitors alone only had partial effects on nuclear TRADD-induced death, but when combined, zVAD.fmk and AEBSF or Boc-D and AEBSF completely inhibited nuclear TRADD-induced apoptosis (Figure $3 \mathrm{c}$ ). These data suggest that caspases are cleaved and activated in response to nuclear TRADD expression, but that another protease also participates in nuclear TRADD-induced death. Similar results were obtained in MEFs using zVAD.fmk and AEBSF (Figure 3c).

\section{Caspase-2 is not the initiator caspase activated by the expression of nuclear TRADD}

Caspase-2 was an attractive candidate for the initiator caspase that is activated by nuclear TRADD for the following reasons: (1) Caspase-2 has been implicated in mediating nuclear stress-induced apoptosis. ${ }^{22}$ (2) Caspase-2 can function upstream of the mitochondria leading to caspase-9 activation. $^{23-25}$ (3) Caspase-2 forms a multiprotein nuclear complex termed the PIDDosome ${ }^{26}$ which involves deathdomain proteins. ${ }^{26}$ In addition, caspase-2 catalytic activity is not efficiently inhibited by zVAD.fmk, ${ }^{27}$ but has been reported to be inhibited by AEBSF. ${ }^{28,29}$ To test if caspase-2 is the initiator caspase that is activated by nuclear TRADD, we depleted caspase-2 in wild-type IMR90E1A cells and IMR90E 1 A cells stably expressing either wild-type caspase-2, or a point mutant of caspase-2 that cannot be degraded using short interfering RNA (siRNA) oligos. ${ }^{22}$ After depletion, one set of cells was microinjected with either YFP or YFP-nuclear TRADD and the second set of cells was harvested for immunoblotting to confirm that caspase-2 depletion occurred. Viability of caspase-2 depleted nuclear TRADD expressing cells was determined at 18-24 $\mathrm{h}$ after injection. The cells were successfully depleted of caspase-2 (Figure $4 \mathrm{a}$, inset); however this did not confer resistance to nuclear TRADD-induced death (Figure 4a).

To eliminate the possibility that residual caspase-2 may be sufficient to activate the nuclear TRADD pathway, we obtained caspase-2 deficient MEFs. ${ }^{30}$ Wild type and cas- 

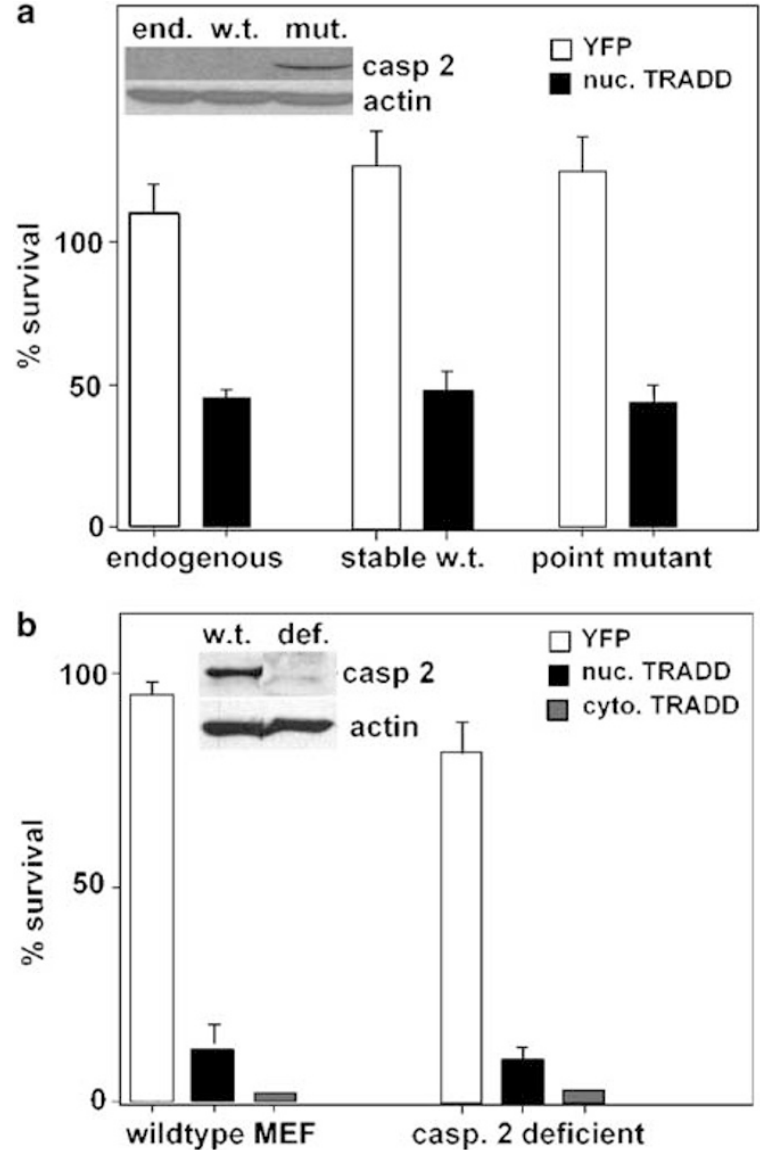

Figure 4 Caspase-2 is not required for nuclear TRADD-induced death. (a) IMR90E1A cells expressing either only endogenous caspase-2, stable wild-type caspase-2, or stable point mutant caspase-2 were transfected with double stranded RNA oligos. At $24 \mathrm{~h}$ after depletion, cells were harvested and whole-cell lysates were blotted with an anti-caspase-2 (inset) or microinjected with either YFP or YFP-nuclear TRADD. Caspase-2 depletion did not inhibit nuclear TRADD-induced cell death. (b) Caspase-2 null MEFs were characterized by Western blotting (inset) and microinjected with YFP, YFP-nuclear TRADD, or YFP-cytoplasmic TRADD and cell survival was assessed 18-24 $\mathrm{h}$ after injection. Both nuclear and cytoplasmic TRADD efficiently killed the caspase-2 deficient cells

pase-2 deficient MEFs were microinjected with YFP, YFP. nuclear TRADD, or YFP-cytoplasmic TRADD. Both cell types were equally sensitive to both nuclear and cytoplasmic TRADD-induced death (Figure 4b) indicating that caspase-2 expression is not required for nuclear TRADD-induced apoptosis. We conclude that caspase-2 is not the initiator caspase activated by nuclear TRADD expression and therefore that the nuclear TRADD protein does not activate the PIDDosome.

\section{Caspase- 9 is required for the activation of nuclear TRADD-induced apoptosis}

Since we eliminated caspase- 2 and caspase-8 as the initiator caspase activated in response to nuclear TRADD expression and because we previously showed that the antiapoptotic protein $\mathrm{Bcl}-\mathrm{XL}$ is able to inhibit nuclear TRADD-induced
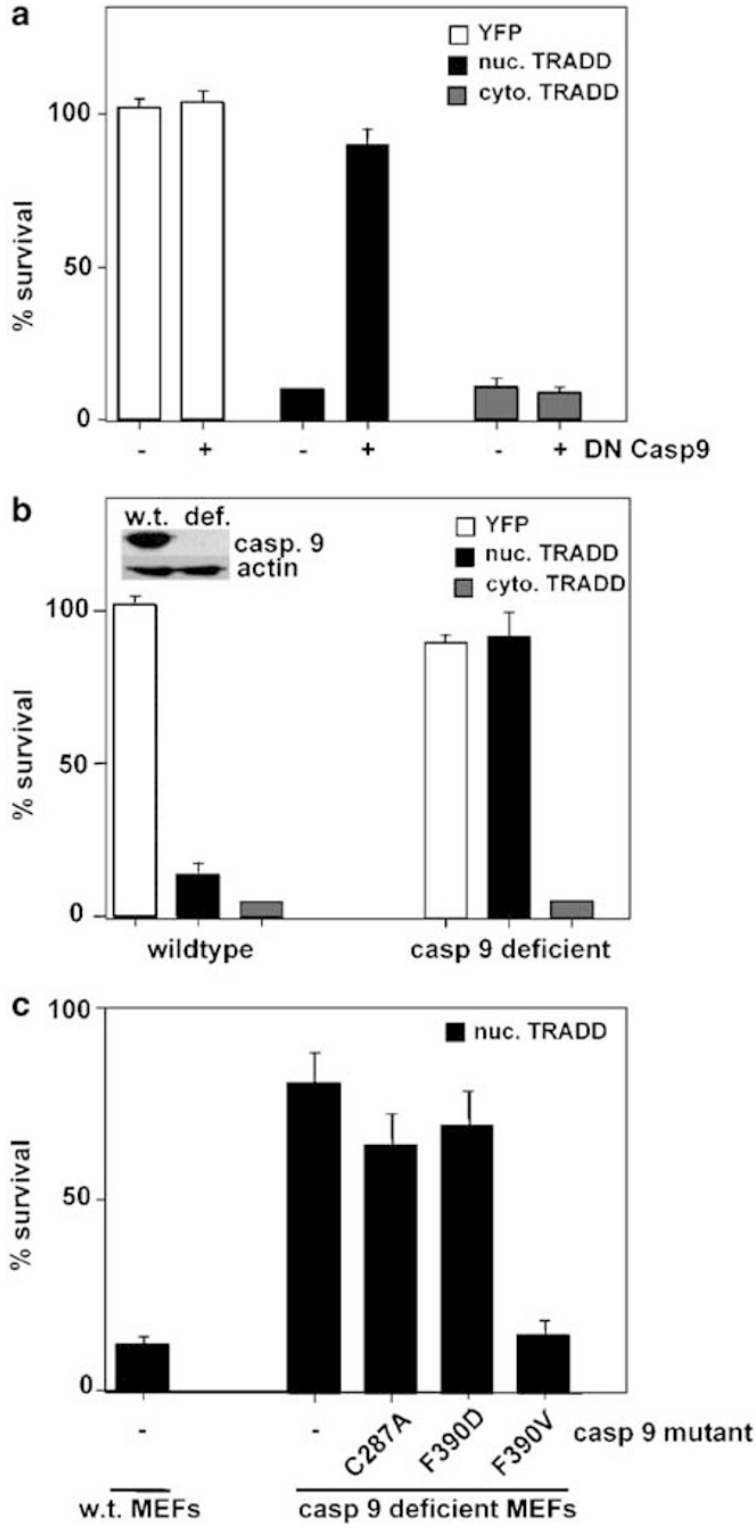

Figure 5 Caspase-9 is required for nuclear TRADD-induced death. (a) HeLa cells were co-injected with YFP, YFP-nuclear TRADD or YFP-cytoplasmic TRADD and either empty vector $(-)$ or a DN-caspase-9 expression plasmid $(+)$. Dominant-negative caspase-9 inhibited death caused by nuclear TRADD but did not affect cytoplasmic TRADD-induced cell death. (b) Wild type and caspase-9 null MEFs were characterized by Western blotting (inset) and microinjected with YFP, YFP-nuclear TRADD, or YFP-cytoplasmic TRADD. Caspase-9-deficient cells were resistant only to nuclear TRADD-induced death. (c) Wildtype or caspase-9 null MEFs were co-injected with YFP-nuclear TRADD and either empty vector (-) or caspase-9 point mutants. Only the F390V point mutant rescued nuclear TRADD-induced cell death indicating that caspase-9 must have an intact catalytic site and dimerization interface to rescue nuclear TRADD-induced cell death

death, ${ }^{18}$ we next asked whether caspase-9, which is activated by nuclear TRADD (Figure 3b), was required for nuclear TRADD-induced death. A catalytically inactive DN caspase-9 construct was co-injected with YFP, nuclear TRADD, or cytoplasmic TRADD. Using this approach, we found that DNcaspase- 9 could completely inhibit death induced by nuclear 
TRADD, but as predicted, had no effect on death induced by cytoplasmic TRADD (Figure 5a), which works through caspase-8 (Figure 3a).

To confirm this finding, the TRADD mutants were microinjected into caspase- 9 wild type or caspase- 9 null MEFs. ${ }^{31}$ Cell viability was assessed $18-24 \mathrm{~h}$ after injection. Caspase- 9 deficiency conferred complete protection from nuclear TRADD-induced death but had no significant effect on cytoplasmic TRADD (Figure 5b). Dimer formation at the apoptosome can drive caspase- 9 activation. ${ }^{32}$ To further define the role of caspase- 9 , we used three caspase- 9 point mutants. $^{32}$ Mutation of the active site cysteine of caspase- 9 (C287A) generates a dimerization competent but catalytically dead caspase-9 molecule. Mutation of the dimerization interface phenylalinine to an aspartic acid residue (F390D) generates a dimerization defective molecule with an intact active site. Mutation of the dimerization interface phenylalinine to a valine (F390V) generates a dimerization competent and catalytically active caspase- 9 molecule with wild-type function. We tested whether expression of any of the caspase9 mutants in the caspase- 9 knockout MEFs could restore sensitivity to nuclear TRADD-induced death. Only the expression of the dimerization competent and catalytically active F390V caspase-9 was able to restore sensitivity to nuclear TRADD-induced death (Figure 5c). These data indicate that caspase-9 expression, dimerization, and catalytic activity are required for nuclear TRADD-induced death.

\section{APAF-1 deficiency confers partial protection from nuclear TRADD-induced apoptosis}

Caspase- 9 activation occurs downstream of mitochondrial cytochrome $c$ release by complexing with APAF-1, dATP, and cytochrome $c$ to form the apoptosome. To clarify the role of apoptosome formation in nuclear TRADD-induced death, we obtained APAF-1 deficient MEFs. ${ }^{33}$ Wild type and APAF-1 deficient MEFs were injected with YFP, YFP-nuclear TRADD, or GFP-cytoplasmic TRADD. Cell survival was assessed at 18-24 $\mathrm{h}$ after injection. We found that APAF-1 deficient MEFs were partially but not completely resistant to nuclear TRADDinduced death suggesting that caspase- 9 was activated at least in part, independently of apoptosome formation (Figure 6a).

Since the APAF-1 deficient MEFs were partially resistant to nuclear TRADD-induced death, we wondered if the APAF-1independent pathway was mediated by the activation of an AEBSF-sensitive protease. If the serine protease-dependent arm is activated independently of APAF-1 expression, then AEBSF should completely protect the APAF-1 deficient MEFs from nuclear TRADD-induced death. Nuclear TRADD was expressed in the APAF-1 null MEFs treated with either zVAD.fmk or AEBSF and counted. Cell viability was assessed after $18-24 \mathrm{~h}$, indicating that zVAD.fmk but not AEBSF cooperated with APAF-1 deficiency to protect MEFs from nuclear TRADD-induced death (Figure 6b). These data suggest that an APAF-1-independent mechanism that induces caspases but not serine proteases contributes to nuclear TRADD-induced death.
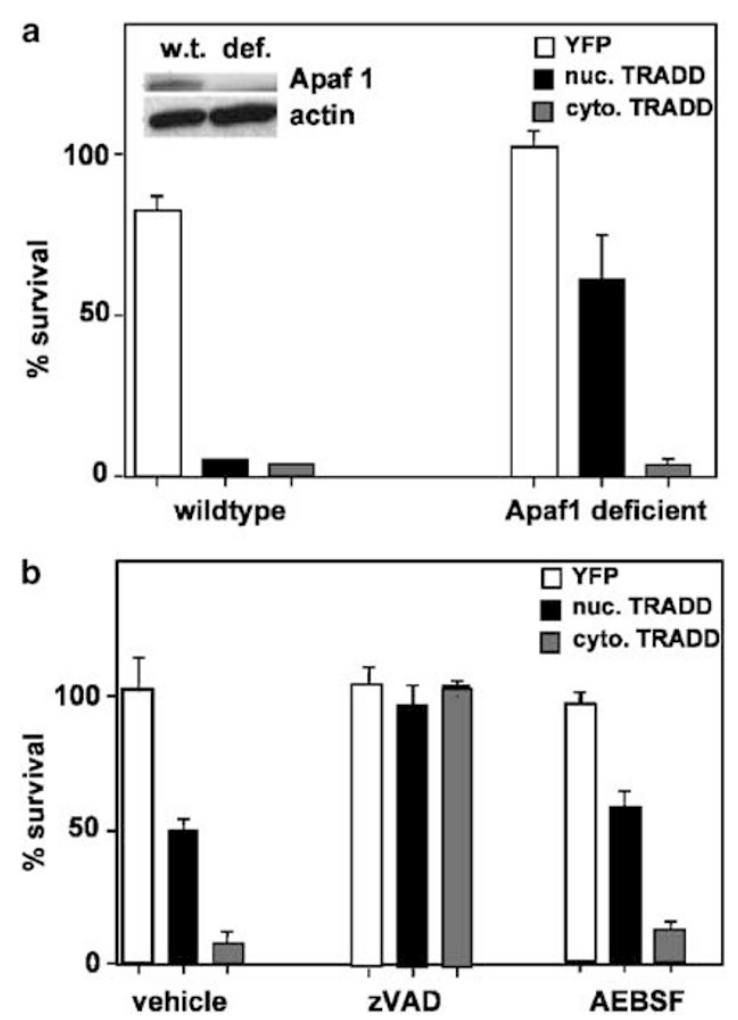

Figure 6 Deficiency in Apaf-1 does not confer complete resistance to nuclear TRADD-induced death. (a) Wild type and APAF-1 null MEFs were characterized by Western blotting (inset) and microinjected with YFP, YFP-nuclear TRADD, or YFP-cytoplasmic TRADD. Apaf-1 deficient cells were partially resistant to nuclear TRADD-induced cell death. (b) Apaf-1 null MEFs were microinjected with YFP, YFP-nuclear TRADD or YFP-cytoplasmic TRADD. At $4 \mathrm{~h}$ after injection, the cells were treated with DMSO, zVAD.fmk, or AEBSF and survival was determined $18 \mathrm{~h}$ later. Complete rescue of nuclear TRADD-induced cell death was obtained by combining Apaf-1 deficiency with caspase inhibition. Cooperation was not obtained with Apaf-1 deficiency and AEBSF. Cytoplasmic TRADD was blocked by caspase inhibition but not by AEBSF

\section{Signal transducer and activator of transcription I (STAT1) is not involved in the nuclear TRADD- induced cell death pathway}

Recent studies by Wesemann et $a^{\beta 4}$ indicate that in response to interferon, TRADD translocates from the cytoplasm to the nucleus and interacts with and regulates STAT-1. We therefore used wild type and STAT-1 deficient cells to assess whether STAT-1 was important in mediating nuclear TRADD induced death. STAT-1 wild-type and deficient cells displayed the same sensitivity to nuclear TRADD indicating that STAT-1 is not required for the activation of this nuclear cell death pathway. Since we previously showed that expression of p53 in deficient cells increases sensitivity to nuclear TRADDinduced cell death, ${ }^{18}$ it is possible that p53 primarily functions upstream of only one arm (i.e. zVAD.fmk- or AEBSFsensitive) of the pathway. We therefore asked if nuclear TRADD-induced death of p53 deficient MEFs, which occurs to a lesser extent than in wild-type cells, was inhibited by either protease inhibitor. Figure $7 \mathrm{~b}$ shows that unlike the situation with wild-type MEFs (Figure 3c) where both zVAD.fmk and AEBSF contribute about equally to protection, the majority of 

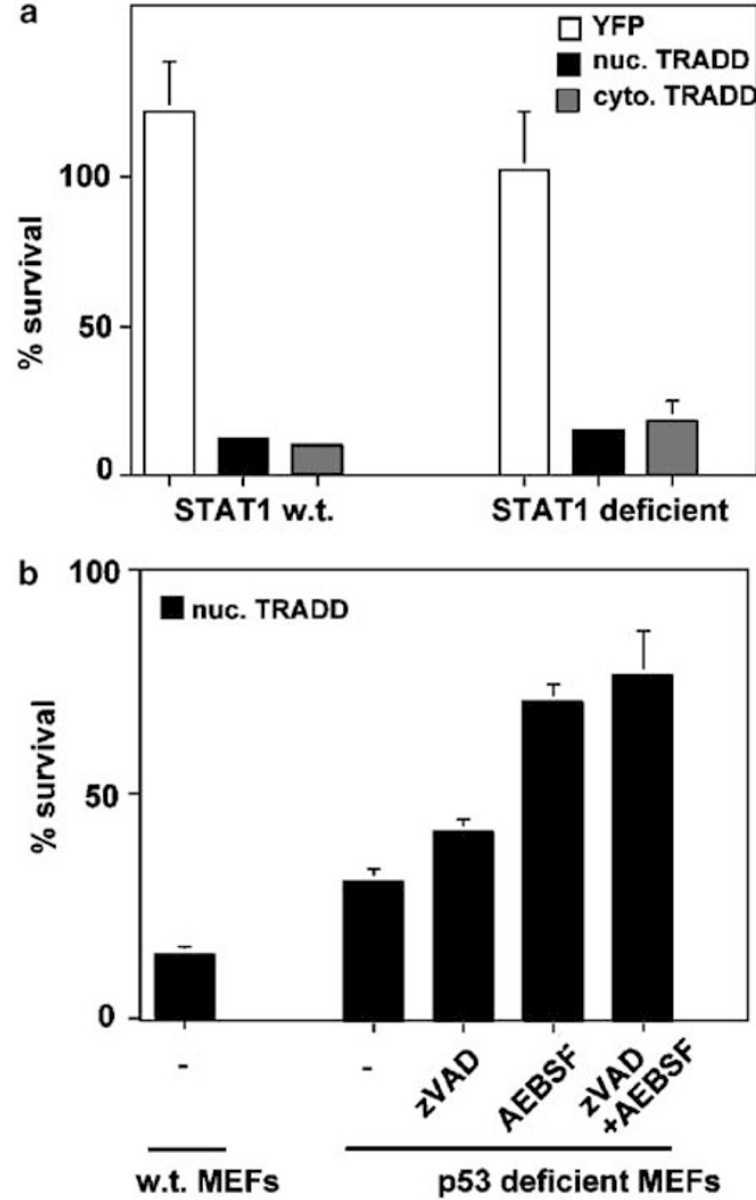

Figure 7 STAT1 deficiency does not alter nuclear TRAFF-induced death while p53 deficiency alters the protease sensitivity of nuclear TRADD-induced death. (a) Wild type or STAT1 deficient cells were microinjected with YFP, YFP-nuclear TRADD, or YFP-cytoplasmic TRADD. Stat-1 deficient cells were equally sensitive to both nuclear and cytoplasmic TRADD. (b) Nuclear TRADD was microinjected into wild type or p53-deficient MEFs then treated with caspase and serine protease inhibitors. p53 deficiency alone reduced the sensitivity to nuclear TRADD and treatment with AEBSF rescued these cells indicating that the p53dependent signal induced by nuclear TRADD works primarily through caspases

the protection was conferred by AEBSF on its own suggesting that most of the p53-dependent signaling occurs through caspases.

\section{Discussion}

In this study we show that TRADD can activate different apoptotic pathways from the nucleus and cytoplasm. Death induced by nuclear TRADD is not blocked by zVAD.fmk on its own, but can be blocked by combined inhibition of caspases and serine proteases. In contrast, a cytoplasmic TRADD protein that is tethered to the membrane and cannot move to the nucleus kills cells through an entirely caspase-dependent mechanism that is activated through the FADD-caspase-8 pathway and can be inhibited by zVAD.fmk alone. This work therefore adds to several recent examples where serine proteases cause caspase-independent apoptosis. For example, Egger et al. ${ }^{35}$ described a serine protease mediated cell death that resembles caspase-dependent apoptosis. This death pathway was inhibited by AEBSF and blocked by $\mathrm{Bcl}-2$ overexpression, characteristics similar to nuclear TRADDinduced death. Another report found that a serine protease was involved in the initiation of DNA damage-induced apoptosis. ${ }^{36}$ There are other examples where death domains activate different kinds of apoptosis pathways. For example, expression of the death domains of two members of the death receptor family from Xenopus laevis (xDR-M1 and xDR-M2) activates two distinct apoptotic pathways. While death induced by expression of the death domain of $x D R-M 2$ is blocked by inhibition of caspase-8, death induced by expression of the death domain of $\mathrm{xDR}-\mathrm{M} 1$ is blocked by serine protease inhibition. $^{37}$

Our data indicate that the apoptotic pathway activated by nuclear TRADD does not require caspase-8 or caspase- 2 . Instead, death is dependent upon caspase-9, which must have an intact catalytic site and be able to form dimers. The most straightforward interpretation of these results is that nuclear TRADD causes mitochondrial disruption that leads to caspase- 9 activation through the apoptosome leading to effector caspase activation. However, our experiments indicate that while this happens, the apoptotic pathway that is induced by the nuclear TRADD molecule is more complex and that while signaling is dependent upon caspase- 9 it may also involve other signaling molecules. This idea is based on the following argument. One might expect that a purely caspase9-dependent cell death pathway would be inhibited by pharmacological caspase inhibition, caspase-9 deficiency, $\mathrm{Bcl}-\mathrm{xL}$ or $\mathrm{Bcl}-2$ expression or disruption of the apoptosome. However, Apaf-1 deficiency or caspase inhibition confers only partial protection against nuclear TRADD whereas caspase- 9 deficiency and $\mathrm{Bcl}-\mathrm{xL}$ expression ${ }^{18}$ confer complete protection. These data suggest that active dimerized caspase- 9 can signal downstream effects in addition to the activation of effector caspases. Other Bcl-2 sensitive cell death pathways that are independent of Apaf-1 have been identified in other systems further supporting this idea. ${ }^{38,39}$ Though APAF-1 deficient cells displayed reduced sensitivity to nuclear TRADD-induced death, the finding that zVAD.fmk but not AEBSF cooperates with APAF-deficiency to confer complete resistance suggests that the AEBSF-sensitive pathway is APAF-1 dependent.

We do not know which signaling molecules mediate nuclear TRADD signaling upstream of caspase-9. One likely candidate was caspase-2, which seems to play no role in the response. We previously found that p53 loss partly protected against nuclear TRADD and that p53 expression could sensitize cells to nuclear TRADD. ${ }^{18}$ In support of this view, p53 deficient MEFs responded slightly differently compared to their wild-type counterparts. The p53-deficient cells died less efficiently than wild-type cells in response to nuclear TRADD and this death was primarily mediated through the AEBSFsensitive signals implying that the sensitization that is conferred by $\mathrm{p} 53$ is mainly upstream of the caspasedependent cell death. PML deficiency also conferred protection to nuclear TRADD. ${ }^{18}$ However, we could not determine if the PML-mediated effects were sensitive to only one of the inhibitors because unlike the situation with p53-/- cells, PML-/- cells displayed no significant nuclear TRADDinduced death compared with control injections. ${ }^{18}$ Although 
STAT1 has been identified as a mediator of nuclear TRADD signaling after interferon treatment, ${ }^{34}$ we found that STAT1deficient cells were equally sensitive to nuclear TRADDinduced death. Thus, although p53 and PML, caspase- 9 and Apaf- 1 are involved in this response, the mechanism by which the cell death signal is mediated upstream and downstream of caspase- 9 is complicated and involves multiple proteins that have both overlapping and separable effects.

TRADD may therefore be able to participate in three distinct complexes through its DD. In the first, which occurs at the cell membrane in association with the activated TNFR1 receptor, TRADD interacts with TNFR1, TRAF proteins, and RIP, and this leads primarily to antiapoptotic signaling. After TNFR1 stimulation, a cytoplasmic complex ${ }^{16}$ or a complex in endocytic vesicles ${ }^{17}$ involving TRADD interactions with FADD leads to caspase-8-dependent apoptosis. Finally a third, nuclear complex that does not involve FADD or caspase-8 can also lead to apoptosis, but this occurs through caspase-9 and leads to both caspase-dependent and caspase-independent effects.

\section{Materials and Methods}

\section{Cell lines}

HeLa cells were maintained according to ATCC. IMR90E1A cells were maintained as described. ${ }^{22}$ Mouse embryonic fibroblasts were maintained in DMEM-high glucose, 10\% FBS, $2 \mathrm{mM}$ L-glutamine. BJAB cells were maintained in RPMI with $10 \%$ FBS.

\section{Immunoblotting}

Cells were harvested in NP-40 lysis buffer. Bio-Rad protein assay was performed and $20-40 \mu$ g protein was loaded onto $12 \%$ SDS-PAGE gel, and transferred onto PVDF. Blots were probed with antiactive caspase-3 antibody (Cell Signaling, Beverly, MA, USA), PARP (Cell Signaling), caspase-9 (Cell Signaling), antiactin antibody (Sigma), anti-caspase-2 antibody (Alexis), and anti-murine APAF-1 (Cell Signaling). BJAB cells were probed with an anti-human caspase-8 (Cell Signaling).

\section{Transient transfections}

HeLa cells were plated to $60-80 \%$ confluency and transiently transfected with $2 \mu \mathrm{g}$ plasmid DNA using the Fugene6 transfection reagent (Roche). Transfection efficiency in HeLa cells averaged between 60 and $80 \%$. BJAB cells and MEFs were plated as instructed by the manufacturer and transfected using lipofectamine reagent (Invitrogen, La Jolla, CA, USA). Transfection efficiency in MEFs was approximately 30-50\%. Caspase assays were performed using a caspase- 9 colorimetric assay (Calbiochem, La Jolla, CA, USA).

\section{Microinjection}

Cells were plated in 35-mm dishes and microinjected using an Eppendorf microinjector on a Zeiss Axiovert 100 microscope with $250 \mu \mathrm{M}$ plasmid DNA in $50 \%$ PBS. Cell viability was determined as previously described ${ }^{40}$ with each successfully injected cell assessed. At 2-4 $\mathrm{h}$ after injection, the fluorescent cells were counted. After 18-24 h, the surviving cells were recounted to generate percent survival. Experiments are representative of at least 250 injected cells per injectate from at least four separate experiments. Survival greater than $100 \%$ indicates cell proliferation during the experiment. zVAD.fmk (Alexis, San Diego, CA, USA) and Boc-D.fmk (Calbiochem) were used at a final concentration of $50 \mu \mathrm{M}$. AEBSF (SigmaAldrich, St. Louis, MO, USA) was used at a final concentration of $300 \mu \mathrm{M}$ in HeLa cells. Due to toxicity caused by AEBSF treatment alone in MEFs, the concentration of AEBSF was reduced to $200 \mu \mathrm{M}$ in these cells.

\section{SiRNA}

Caspase-2 was depleted in IMR90E1a cells as described. ${ }^{22}$ At 24 and $48 \mathrm{~h}$ after depletion cells were harvested for immunoblotting and injected with YFP-nuclear TRADD or YFP. Percent survival was calculated as described above.

\section{Directed 2 hybrid}

FADD interactions with full-length TRADD or the truncated nuclear TRADD construct were performed as previously described. ${ }^{41}$

\section{Acknowledgements}

We thank Drs. T Mak, J Yuan, G Stark, G Salvesen, and Y Lazebnik for providing critical cells and constructs. This work was supported by $\mathrm{NIH}$ Grant NS46353 (AT).

\section{References}

1. Hengartner MO (2000) The biochemistry of apoptosis. Nature 407: 770-776

2. Budihardjo I, Oliver H, Lutter M, Luo X and Wang X (1999) Biochemical pathways of caspase activation during apoptosis. Annu. Rev. Cell Dev. Biol. 15: 269-290

3. Ashkenazi A and Dixit VM (1999) Apoptosis control by death and decoy receptors. Curr. Opin. Cell Biol. 11: 255-260

4. Thorburn A (2004) Death receptor-induced cell killing. Cell. Signal. 16: $139-144$

5. Luo X, Budihardjo I, Zou H, Slaughter C and Wang X (1998) Bid, a Bcl2 interacting protein, mediates cytochrome $c$ release from mitochondria in response to activation of cell surface death receptors. Cell 94: 481-490

6. Leist M and Jaattela M (2001) Four deaths and a funeral: from caspases to alternative mechanisms. Nat. Rev. Mol. Cell Biol. 2: 589-598

7. Johnson DE (2000) Noncaspase proteases in apoptosis. Leukemia 14: $1695-1703$

8. Leist M and Jaattela M (2001) Triggering of apoptosis by cathepsins. Cell Death Differ. 8: 324-326

9. Martins LM, laccarino I, Tenev T, Gschmeissner S, Totty NF, Lemoine NR, Savopoulos J, Gray CW, Creasy CL, Dingwall C and Downward J (2002) The serine protease Omi/HtrA2 regulates apoptosis by binding XIAP through a Reaper-like motif. J. Biol. Chem. 277: 439-444

10. Hegde R, Srinivasula SM, Zhang Z, Wassell R, Mukattash R, Cilenti L, DuBois G, Lazebnik Y, Zervos AS, Fernandes-Alnemri T and Alnemri ES (2002) Identification of Omi/HtrA2 as a mitochondrial apoptotic serine protease that disrupts IAP-caspase interaction. J. Biol. Chem. 277: 432-438

11. van Loo G, van Gurp M, Depuydt B, Srinivasula SM, Rodriguez I, Alnemri ES Gevaert K, Vandekerckhove J, Declercq W and Vandenabeele P (2002) The serine protease $\mathrm{Omi} / \mathrm{HtrA} 2$ is released from mitochondria during apoptosis. Omi interacts with caspase-inhibitor XIAP and induces enhanced caspase activity. Cell Death Differ. 9: 20-26

12. Srinivasula SM, Gupta S, Datta P, Zhang Z, Hegde R, Cheong N, FernandesAlnemri T and Alnemri ES (2003) Inhibitor of apoptosis proteins are substrates for the mitochondrial serine protease Omi/HtrA2. J. Biol. Chem. 278: $31469-31472$ 
13. Yang QH, Church-Hajduk R, Ren J, Newton ML and Du C (2003) Omi/HtrA2 catalytic cleavage of inhibitor of apoptosis (IAP) irreversibly inactivates IAPs and facilitates caspase activity in apoptosis. Genes Dev. 17: 1487-1496

14. Chen G and Goeddel DV (2002) TNF-R1 signaling: a beautiful pathway. Science. 296: 1634-1635

15. Wajant $H$, Pfizenmaier $K$ and Scheurich $P$ (2003) Tumor necrosis factor signaling. Cell Death Differ. 10: 45-65

16. Micheau $\mathrm{O}$ and Tschopp J (2003) Induction of TNF receptor I-mediated apoptosis via two sequential signaling complexes. Cell 114: 181-190

17. Schneider-Brachert W, Tchikov V, Neumeyer J, Jakob M, Winoto-Morbach S, Held-Feindt J, Heinrich M, Merkel O, Ehrenschwender M, Adam D, Mentlein R Kabelitz D and Schutze S (2004) Compartmentalization of TNF receptor 1 signaling: internalized TNF receptosomes as death signaling vesicles. Immunity 21: 415-428

18. Morgan M, Thorburn J, Pandolfi PP and Thorburn A (2002) Nuclear and cytoplasmic shuttling of TRADD induces apoptosis via different mechanisms. J. Cell Biol. 157: 975-984

19. Gomez-Angelats M and Cidlowski JA (2003) Molecular evidence for the nuclear localization of FADD. Cell Death Differ. 10: 791-797

20. Screaton RA, Kiessling S, Sansom OJ, Millar CB, Maddison K, Bird A, Clarke AR and Frisch SM (2003) Fas-associated death domain protein interacts with methyl-CpG binding domain protein 4: a potential link between genome surveillance and apoptosis. Proc. Natl. Acad. Sci. USA 100: 5211-5216

21. Hsu H, Shu H-B, Pan M-G and Goeddel DV (1996) TRADD-TRAF2 and TRADD-FADD interactions define two distinct TNF receptor 1 signal transduction pathways. Cell 84: 299-308

22. Lassus $P$, Opitz-Araya $X$ and Lazebnik $Y$ (2002) Requirement for caspase-2 in stress-induced apoptosis before mitochondrial permeabilization. Science 297 1352-1354

23. Paroni G, Henderson C, Schneider C and Brancolini C (2002) Caspase-2 can trigger cytochrome $c$ release and apoptosis from the nucleus. J. Biol. Chem. 277: 15147-15161

24. Guo Y, Srinivasula SM, Druilhe A, Fernandes-Alnemri T and Alnemri ES (2002) Caspase-2 induces apoptosis by releasing proapoptotic proteins from mitochondria. J. Biol. Chem. 277: 13430-13437

25. Troy CM and Shelanski ML (2003) Caspase-2 redux. Cell Death Differ. 10 $101-107$

26. Tinel A and Tschopp J (2004) The PIDDosome, a protein complex implicated in activation of caspase-2 in response to genotoxic stress. Science 304: 843-846

27. Garcia-Calvo M, Peterson EP, Leiting B, Ruel R, Nicholson DW and Thornberry NA (1998) Inhibition of human caspases by peptide-based and macromolecular inhibitors. J. Biol. Chem. 273: 32608-32613

28. Stefanis L, Troy CM, Qi H, Shelanski ML and Greene LA (1998) Caspase-2 (Nedd-2) processing and death of trophic factor-deprived PC12 cells and sympathetic neurons occur independently of caspase-3 (CPP32)-like activity. J. Neurosci. 18: 9204-9215
29. Stefanis L, Troy CM, Qi H and Greene LA (1997) Inhibitors of trypsin-like serine proteases inhibit processing of the caspase Nedd-2 and protect PC12 cells and sympathetic neurons from death evoked by withdrawal of trophic support. J. Neurochem. 69: 1425-1437

30. Bergeron L, Perez Gl, Macdonald G, Shi L, Sun Y, Jurisicova A, Varmuza S, Latham KE, Flaws JA, Salter JC, Hara H, Moskowitz MA, Li E, Greenberg A, Tilly $\mathrm{JL}$ and Yuan $\mathrm{J}$ (1998) Defects in regulation of apoptosis in caspase-2deficient mice. Genes Dev. 12: 1304-1314

31. Hakem R, Hakem A, Duncan GS, Henderson JT, Woo M, Soengas MS, Elia A, de la Pompa JL, Kagi D, Khoo W, Potter J, Yoshida R, Kaufman SA, Lowe SW, Penninger JM and Mak TW (1998) Differential requirement for caspase 9 in apoptotic pathways in vivo. Cell 94: 339-352

32. Renatus M, Stennicke HR, Scott FL, Liddington RC and Salvesen GS (2001) Dimer formation drives the activation of the cell death protease caspase 9 . Proc. Natl. Acad. Sci. USA 98: 14250-14255

33. Yoshida H, Kong YY, Yoshida R, Elia AJ, Hakem A, Hakem R, Penninger JM and Mak TW (1998) Apaf1 is required for mitochondrial pathways of apoptosis and brain development. Cell 94: 739-750

34. Wesemann DR, Qin H, Kokorina N and Benveniste EN (2004) TRADD interacts with STAT1-alpha and influences interferon-gamma signaling. Nat. Immunol. 5: 199-207

35. Egger L, Schneider J, Rheme C, Tapernoux M, Hacki J and Borner C (2003) Serine proteases mediate apoptosis-like cell death and phagocytosis under caspase-inhibiting conditions. Cell Death Differ. 10: 1188-1203

36. De Bruin EC, Meersma D, De Wilde J, Den Otter I, Schipper EM, Medema JP and Peltenburg LT (2003) A serine protease is involved in the initiation of DNA damage-induced apoptosis. Cell Death Differ. 10: 1204-1212

37. Tamura K, Noyama T, Ishizawa YH, Takamatsu N, Shiba T and Ito M (2004) Xenopus death receptor-M1 and -M2, new members of the tumor necrosis factor receptor superfamily, trigger apoptotic signaling by differential mechanisms. J. Biol. Chem. 279: 7629-7635

38. Marsden VS, O'Connor L, O'Reilly LA, Silke J, Metcalf D, Ekert PG, Huang DC, Cecconi F, Kuida K, Tomaselli KJ, Roy S, Nicholson DW, Vaux DL, Bouillet P, Adams JM and Strasser A (2002) Apoptosis initiated by Bcl-2-regulated caspase activation independently of the cytochrome c/Apaf- $1 /$ caspase- 9 apoptosome. Nature 419: 634-637

39. Marsden VS, Ekert PG, Van Delft M, Vaux DL, Adams JM and Strasser A (2004) Bcl-2-regulated apoptosis and cytochrome $c$ release can occur independently of both caspase-2 and caspase-9. J. Cell Biol. 165: $775-780$

40. Thorburn J, Bender LM, Morgan MJ and Thorburn A (2003) Caspase- and serine protease-dependent apoptosis by the death domain of FADD in normal epithelial cells. Mol. Biol. Cell 14: 67-77

41. Thomas L, Stillman D and Thorburn A (2002) Regulation of Fas-associated death domain interactions by the death effector domain identified by a modified reverse two hybrid screen. J. Biol. Chem. 277: 34343-34348 